\title{
Generation and molecular characteristics of a highly attenuated GPV strain through adaptation in GEF cells
}

Hongxia Shao ${ }^{1,2,3,4^{*}+}$, Yuchen Jiang ${ }^{1,2,3,4 \dagger}$, Huisha Yuan ${ }^{1,2,3,4}$, Lifei Ji ${ }^{1,2,3,4}$, Wenjie Jin ${ }^{1,2,3,4}$, Kun Qian ${ }^{1,2,3,4}$, Jianqiang Ye $\mathrm{e}^{1,2,3,4}$ and Aijian Qin ${ }^{1,2,3,4^{*}}$

\begin{abstract}
Background: Goose parvoviruses (GPVs) spread globally and cause a huge economic loss to the poultry industry. Although the attenuated GPV vaccines play a key role in preventing the disease caused by GPV, the molecular basis for the attenuation of GPV is barely known.

Results: A highly attenuated GPV strain, GPV-CZM-142, was generated through blindly passaging of the highly pathogenic strain, GPV-CZM, in goose embryonic fibroblasts (GEF) for 142 generations. The GEF-adapted GPV strain's virulence was 10,000 times weaker than its wild type counterpart, GPV-CZM, based on the ELD 50 (50\% Embryo Lethal Dose). By comparing with the wild type strain, genome sequencing analysis identified adapted mutations either in ITR or in NS and VP1 of GPV-CZM-142.
\end{abstract}

Conclusions: The highly attenuated GPV strain, GPV-CZM-142, provides a GPV vaccine candidate, and the identified virulence-related mutations give a novel insight into the molecular determinants of GPV virulence.

Keywords: Goose parvovirus, Attenuated virus, Gene mutation, Vaccine, Virulence

\section{Background}

Goose parvovirus (GPV) belongs to the genus parvoviruses, family Parvoviridae. GPV is a non-enveloped and single-stranded DNA virus. The genome of GPV contains two inverted terminal repeats (ITRs) and two major open reading frames (ORFs). ITR carries important elements for viral replication and encapsidation. The two major ORFs encode the non-structural protein (NS) and the capsid proteins (VP1/2/3). VP1 is the largest capsid protein, which shares a common region of $\mathrm{C}$ terminus with VP2 and VP3. As a globally spread virus,

\footnotetext{
*Correspondence: hxshao@yzu.edu.cn; aijian@yzu.edu.cn

${ }^{\dagger}$ Hongxia Shao and Yuchen Jiang contributed equally to this work.

${ }^{1}$ Key Laboratory of Jiangsu Preventive Veterinary Medicine, Key Laboratory for Avian Preventive Medicine, Ministry of Education, College of Veterinary Medicine, Yangzhou University, No.12 East Wenhui Road, Yangzhou 225009, Jiangsu, China

Full list of author information is available at the end of the article
}

GPV was first isolated and identified in 1956 by Fang in China $[1,2]$. Its infection mainly causes acute contagious and septic diseases in goslings and Muscovy ducks with age younger than $30 \mathrm{~d}$ old [3]. The intestinal suppository is a characteristic lesion for GPV infection in goslings [4]. Although the attenuated GPV vaccine and its yolk antibody play a key role in preventing disease deterioration caused by GPV, GPV is still frequently isolated in the GPV-vaccinated goose. Also, the current strategies for GPV prevention and control are challenged with the following problems: later onset of GPV infection in goose/duck individuals, more emergence of the GPV mutants, and the co-infection of GPV with other pathogens (e.g., goose astrovirus). However, the molecular basis for virulence of GPV remains unclear. In this study, a highly pathogenic GPV strain, GPV-CZM, was continuously passaged in the GEF for 142 generations to

(c) The Author(s). 2020 Open Access This article is licensed under a Creative Commons Attribution 4.0 International License, which permits use, sharing, adaptation, distribution and reproduction in any medium or format, as long as you give appropriate credit to the original author(s) and the source, provide a link to the Creative Commons licence, and indicate if changes were made. The images or other third party material in this article are included in the article's Creative Commons licence, unless indicated otherwise in a credit line to the material. If material is not included in the article's Creative Commons licence and your intended use is not permitted by statutory regulation or exceeds the permitted use, you will need to obtain permission directly from the copyright holder. To view a copy of this licence, visit http://creativecommons.org/licenses/by/4.0/ The Creative Commons Public Domain Dedication waiver (http://creativecommons.org/publicdomain/zero/1.0/) applies to the data made available in this article, unless otherwise stated in a credit line to the data. 
generate a highly attenuated GPV strain, GPV-CZM142 , followed by identification mutations responsible for the attenuation by genomic sequencing analysis.

\section{Results \\ Highly attenuated GPV-CZM-142 generated through adaptation to GEF}

To generate an attenuated GPV vaccine candidate, a highly pathogenic GPV strain GPV-CZM was continuously passaged in GEFs underwent a total of 142 generations. To evaluate the pathogenicity of the adapted GPV-CZM, the ELD 50 of wild type GPV-CZM, the 70th generation of GPV-CZM-70, and the 142nd generation of GPV-CZM-142 were tested in goose embryos. The goose embryos' mortalities infected with the wild type GPV-CZM were $100,100,80,60$, and $40 \%$ at the infection dose of $1: 10,1: 10^{2}, 1: 10^{3}, 1: 10^{4}$, and $1: 10^{5}$ dilutions, respectively. In contrast, those of the goose embryos infected with GPV-CZM-70 were 20, 0, 0, 0 and $0 \%$, respectively. Notably, GPV-CZM-142 was not lethal to goose embryos at these doses. Based on the viral $\mathrm{TCID}_{50}$ (50\% Tissue Culture Infectious Dose) titers of these GPV strains, The ELD 50 of GPD-CZM, GPVCZM-70, and GPV-CZM-142 were $1.46 \mathrm{TCID}_{50}, 10^{5}$ $\mathrm{TCID}_{50}$, and $>10^{5} \mathrm{TCID}_{50}$, respectively (Table 1 ). These data demonstrate that the GPV-CZM-70 and GPVCZM-142 strains are highly attenuated compared with the wild type GPV-CZM.

Sketch on the genome sequences of different GPV strains To profile sequence mutations in the genome of the GEF adapted GPV-CZM strains, the genomes of the GPV-CZM, GPV-CZM-70, and GPV-CZM-142 strains were amplified using primers listed in Table 2. As shown in Fig. 1, six fragments with the expected sizes were efficiently amplified. These amplicons were cloned into the pGEM ${ }^{\circ}-\mathrm{T}$ Easy vector, followed by sequencing the recombinant plasmids. The whole-genome sizes of GPVCZM, GPV-CZM-70, and GPV-CZM-142 were 5106 bp, $5120 \mathrm{bp}$, and $5128 \mathrm{bp}$, respectively. The sequence of GPV-CZM, GPV-CZM-70, and GPV-CZM-142 had been submitted to the GenBank (the accession number for GPV-CZM, GPV-CZM-70, and GPV-CZM-142 was MT939902, MT939903, and MT939904, respectively). Alignment analysis of the genomes of the adapted GPV-

Table 1 Detection of the ELD 50 of GPV-CZM-70 and GPV-CZM142

\begin{tabular}{|c|c|c|c|}
\hline \multirow[t]{2}{*}{ Virus name } & \multicolumn{3}{|l|}{ Virus titer } \\
\hline & $\mathrm{TCID}_{50} / \mathrm{ml}$ & $\mathrm{ELD}_{50} / \mathrm{ml}$ & $\mathrm{TCID}_{50} / \mathrm{ELD}_{50}$ \\
\hline GPV-CZM & $1.58 \times 10^{5}$ & $1.08 \times 10^{5}$ & 1.46 \\
\hline GPV-CZM-70 & $5 \times 10^{5}$ & 5 & $10^{5}$ \\
\hline GPV-CZM-142 & $5 \times 10^{5}$ & $<5$ & $>10^{5}$ \\
\hline
\end{tabular}

CZM-70 and GPV-CZM-142 against the wild type GPVCZM identified several adapted sites in the ITR region. Moreover, 60 single nucleotide mutations in the genome of GPV-CZM-142 compared with the genome of GPVCZM. Among these mutations, 13, 16, and 31 sites were located in the non-coding region, the NS gene, and the VP gene. Genome phylogenic analysis indicated that GPV-CZM, GPV-CZM-70, and GPV-CZM-142 strains were clustered into the same branch as the strains GDaGPV, SYG61v, GPV-98E, and GPV-98D15 (Fig. 2). It is noteworthy that GPV-98D15 is an attenuated GPV through passaging in the duck embryo. The GPV reference strains used for phylogenic analysis were listed in Table 3.

\section{Mutations identified in ITR of GPV-CZM-142}

Since ITR plays vital roles in gene expression and viral replication, ITR of the adapted GPV-CZM-70 and GPVCZM-142 were further analyzed. Although no deletion or insertion was found in the ITR of GPV-CZM-70 and GPV-CZM-142 in comparison with that of the wild type GPV-CZM, the homology of these ITRs from GPVCZM, GPV-CZM-70, and GPV-CZM-142 was 97.198.9\%. As shown in Fig. 3, mutations G32C, G233A, T381C, T382G and A401T were found in the ITR of GPV-CZM-70, and mutations G32C, G44T, C66T, C85T, A100G, T151G, G233A, A256C, G341C, G380A, T381C, T382G and A401T were found in the ITR of GPV-CZM-70 when compared with the ITR of the wild type GPV-CZM.

\section{Mutations identified in NS and VP1 of GPV-CZM-142}

Although no deletion and insertion were identified in the non-structural protein NS and the structural protein VP1 of GPV-CZM-70 and GPV-CZM-142 compared with the wild type strain of GPV-CZM, some singlenucleotide mutations were identified in the proteinencoding genes for both two strains (Tables 4 and 5). Compared with GPV-CZM, GPV-CZM-70 had 18 mutations for an amino acid with 2 mutations located in the NS gene (Table 4) and the other mutations located in the VP1 gene (Table 5). In contrast, GPV-CZM-142 carried 33 mutations for an amino acid with 8 mutations located in the NS gene (Table 4) and the other mutations located in the VP1 gene (Table 5). Notably, of these mutations, 7 and 16 mutations in NS and VP1 were common to GPV-CZM-70 and GPV-CZM-142, respectively. Besides, 7 and 6 mutations in NS and VP1 of GPV-CZM-142 were also found in GPV strain SYG61v, an attenuated vaccine strain. These findings suggest that these mutations are involved in host adaptation of the virus. 
Table 2 Primers used for GPV DNA amplification

\begin{tabular}{|c|c|c|}
\hline Primer name & Primer sequence $\left(5^{\prime}-3^{\prime}\right)$ & Position of the fragment \\
\hline $\mathrm{F} 1$ & TTCAGCTGCTCATTGGAGGGTT & \multirow{2}{*}{$\begin{array}{l}1-203 \\
(4903-5106)\end{array}$} \\
\hline R1 & TTCTCGAGGCGTGGTCAACCTAACA & \\
\hline F2 & CGCATGCCGCGCGGTCAGCCCAAT & \multirow[t]{2}{*}{ 196-1013 } \\
\hline R2 & TATGTATGCTGCAGTCACGGTCTT & \\
\hline F3 & TAAGACCGTGACTGCAGCATAC & \multirow[t]{2}{*}{$1009-2051$} \\
\hline R3 & TTCGCTCGTTCAGGAACGGGCTCTG & \\
\hline F4 & CAGAGCCCGTTCCTGAACGAG & \multirow[t]{2}{*}{$2022-3041$} \\
\hline R4 & CAGAGCCCGTTCCTGAACGAG & \\
\hline F5 & CGAACCTGTGGCAGCATCTGAAATG & \multirow[t]{2}{*}{$3010-4099$} \\
\hline R5 & GTCACTTATTCCTGCTGTAGTGCTG & \\
\hline F6 & CAGCACTACAGCAGGAATAAG & \multirow[t]{2}{*}{$4072-4911$} \\
\hline R6 & CGCATGCGCGCGGTCAGCCCAATAG & \\
\hline
\end{tabular}

\section{Discussion}

The live attenuated GPV vaccine plays a critical role in preventing the disease caused by GPV. However, the frequent emergence of the GPV mutants in the vaccinated goose in China is challenging the current GPV controlling strategies, and the molecular basis for the attenuation of the highly pathogenic GPV needs to be elucidated. In this study, a highly attenuated GPV strain, GPV-CZM-142, was generated by blind passing a highly pathogenic strain GPV-CZM in GEFs. According to the viral titers of $\mathrm{TCID}_{50}$ and $\mathrm{ELD}_{50}$, the virulence of the host-adapted GPV-CZM-142 in GEF was more than 10, 000 times weaker than that of the wild type GPV-CZM. Sequencing analysis revealed adapted mutations through the genome, however, no deletion and insertion were found in genome of GPV-CZM-70 and GPV-CZM-142. Notably, 13 mutations detected in the ITR of GPVCZM-142 covered all the five mutations found in ITR of GPV-CZM-70, indicating the host adaptation of these five mutations in GPV-CZM-70. The previous study indicates that the deletion in the ITR may play roles in the attenuation of the GPV through host adaptation, and GPV strains with short ITR are thought to be less pathogenic [5]. Recently, Zhang et al. also report several insertions in the ITR of the attenuated GPV strains through adaptation in GEF and DEF compared with the wild type strain [6]. However, the roles of these deletions, insertions or,

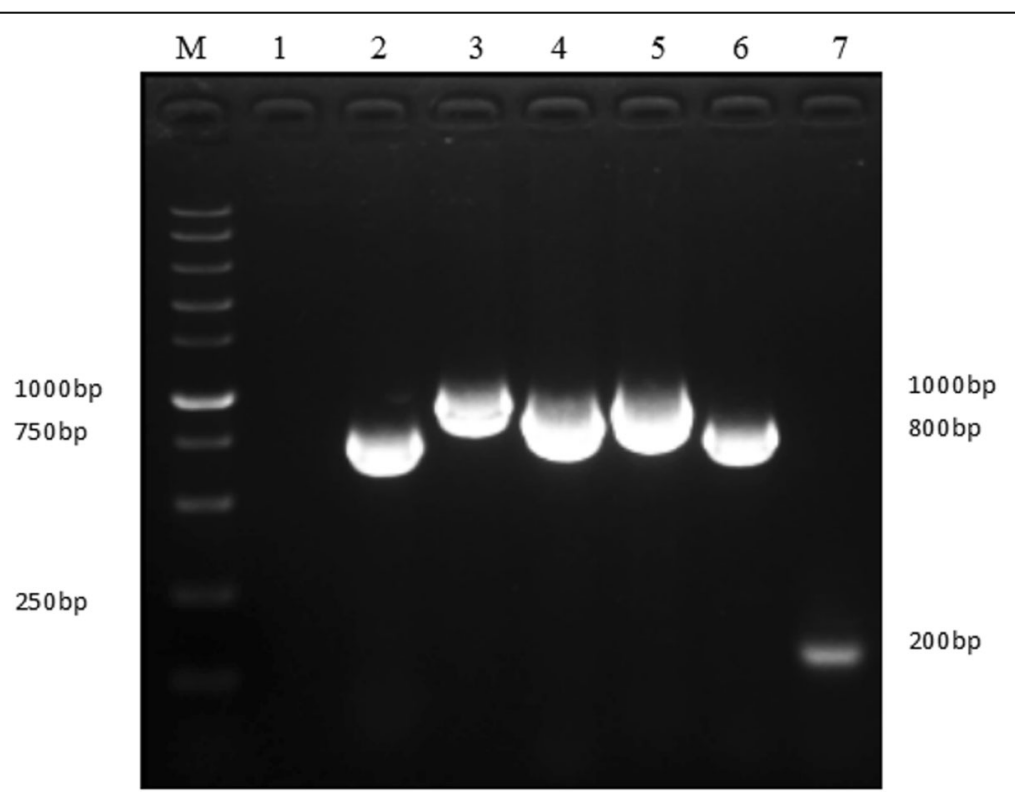

Fig. 1 PCR amplification for the genome of GPV. Lane M. 1 kb Marker; Lane 1. Negative control; Lane 2-7. PCR fragments using primers F2/R2, F3/ R3, F4/R4, F5/R5, F6/R6, and F1/R1, respectively 


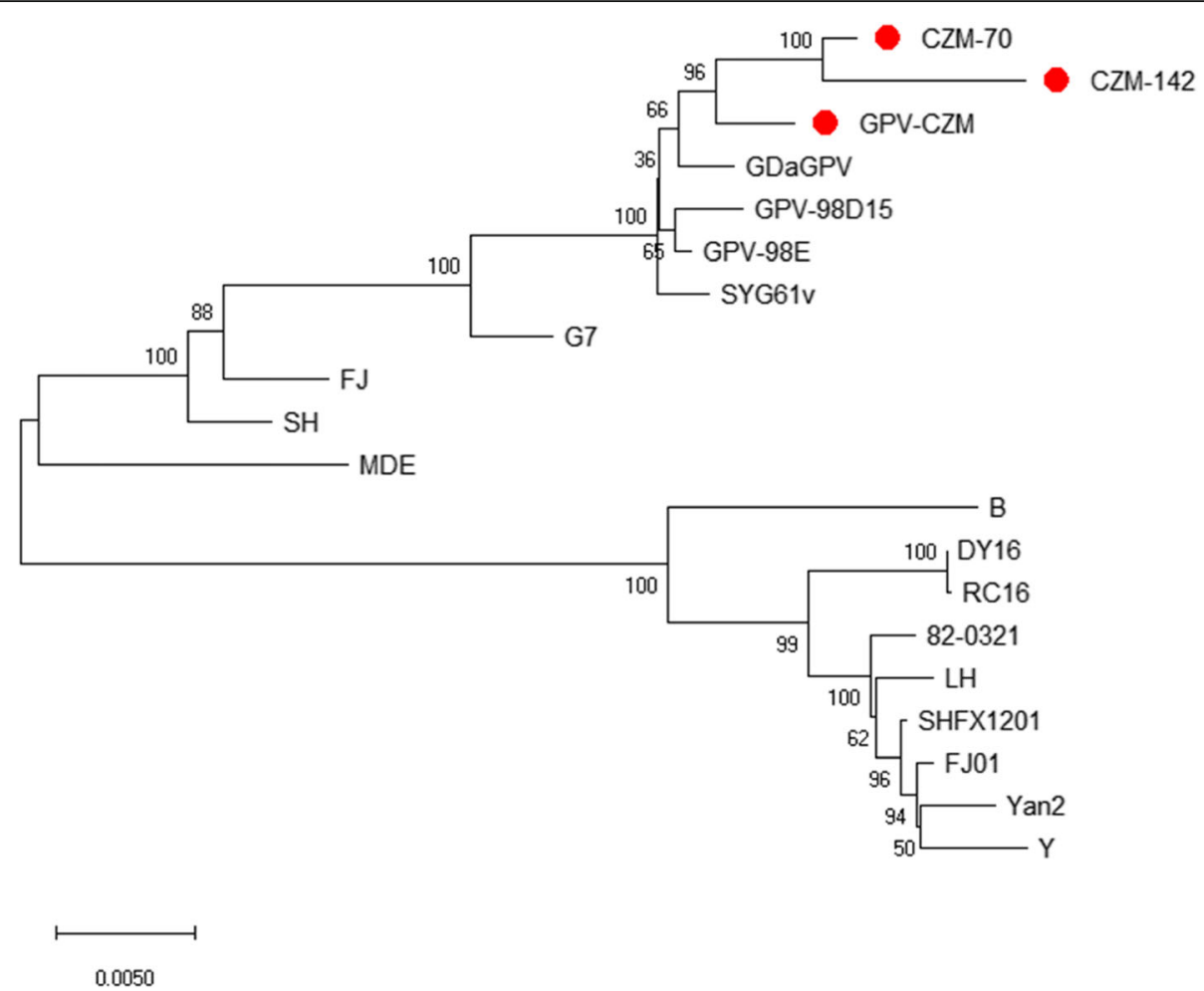

Fig. 2 A phylogenetic tree for the genome of GPV-CZM, GPV-CZM-70, and GPV-CZM-142 was constructed using the neighbor-joining method (1000 bootstraps) with MEGA6

Table 3 The information for 17 GPV reference strains used in this study

\begin{tabular}{llll}
\hline Strain & Genome/bp & Source & Login ID \\
\hline GPV-98E & 5106 & Heilongjiang Province, China & KT598506 \\
GPV-98D15 & 5114 & Heilongjiang Province, China & KT598505 \\
SYG61V & 5102 & Jiangsu Province, China & KC996729 \\
DY16 & 5046 & Jiangsu Province, China & MH209633 \\
LH & 5047 & Jiangsu Province, China & KM272560 \\
SH & 5106 & Shanghai, China & JF333590 \\
SHFX1201 & 5050 & Shanghai Province, China & KC478066 \\
Y & 5106 & Anhui Province, China & KC178571 \\
Yan2 & 5106 & Anhui Province, China & KR136258 \\
GDaGPV & 5106 & Fujian Province, China & HQ891825 \\
G7 & 5106 & Fujian Province, China & KR029617 \\
FJ & 5049 & Fujian Province, China & KY511292 \\
FJ01 & 5104 & Fujian Province, China & KT232256 \\
MDE & 5106 & Fujian Province, China & MF438102 \\
RC16 & 5046 & Sichuan Province, China & KY475562 \\
$82-0321$ & 5050 & Taiwan Province, China & EU583390 \\
B & 5106 & Hungary & U25749 \\
\hline
\end{tabular}

mutations identified here in the attenuation of GPV, need to be further investigated.

Although no insertion or deletion was found in the coding regions in GPV-CZM-70 and GPV-CZM-142 compared with the wild type strain, several mutations were identified in the NS, and VP1 protein-encoding genes in the two adapted GPV strains. As a nonstructural protein, NS plays a vital role in initiating the viral replication and inducing apoptosis in the infected cells [7-9]. VP1, one of the major structural proteins, is thought to bind the cell receptor to mediate the infection of GPV and play critical roles in the pathogenesis of GPV. Recently, a rescuing assay of the recombinant GPV with different VP1 genes by Wang et al. indicates that the chimeric virus with VP1 derived from the virulent strain was pathogenic to goslings, whereas that with VP1 derived from the attenuated strain was non-pathogenic to goslings [10]. Liu et al. also report that $164 \mathrm{~K}, 165 \mathrm{~K}$, and $167 \mathrm{~K}$ of VP1 were vital for the proliferation of GPV in vitro [11]. It is noteworthy that 7 mutations in NS and 16 mutations in VP1 were common to GPV-CZM-70 and GPV-CZM-142 compared with GPV-CZM. Interestingly, 7 mutations in NS and 6 mutations in VP1 of GPV-CZM-142 are also found in an attenuated vaccine strain, SYG61v. All these findings suggest that 


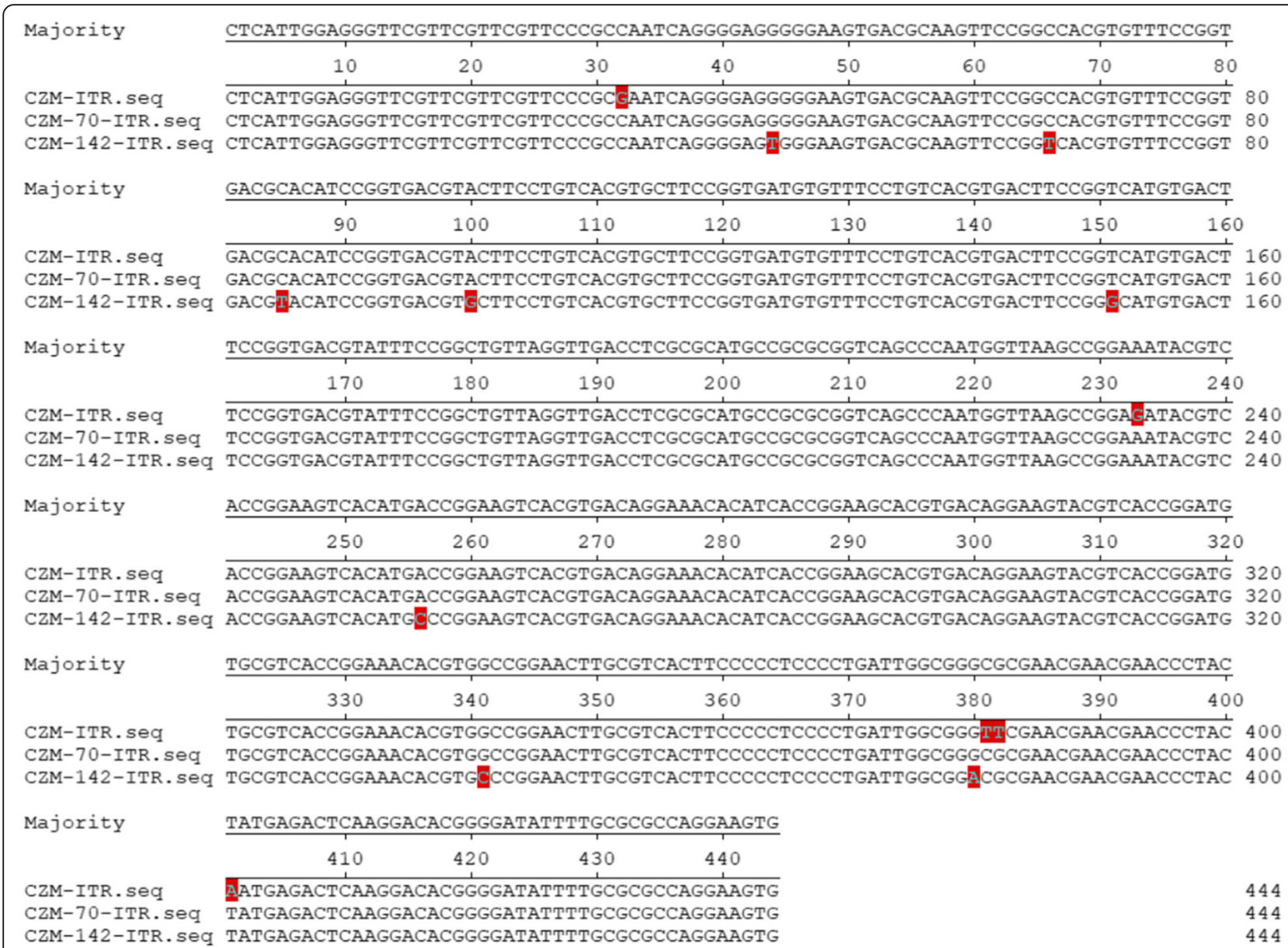

Fig. 3 Adapted sites in the ITR region of GPV-CZM-70 and GPV-CZM-142. The ITR region of GPV-CZM, GPV-CZM-70, and GPV-CZM-142 was aligned, and several adapted sites were identified in ITR of GPV-CZM-70 and GPV-CZM-142, respectively, compared with that of the wild type GPV-CZM

the mutations may contribute to the attenuation of the adapted GPV strains. Moreover, several mutations, such as K532Q, I534M, I537L, R555M, and D594E, in the VP1 found in GPV-CZM-70 and GPV-CZM-142 were located in or very close to the cell receptor

Table 4 Mutations in NS protein of GPV-CZM-70 and GPV-CZM142

\begin{tabular}{lllll}
\hline Position & GPV-CZM & CZM-70 & CZM-142 & SYG61v \\
\hline 72 & I & V & V & I \\
149 & Q & Q & L & Q \\
199 & E & E & D & E \\
203 & A & A & S & A \\
501 & L & L & P & P \\
507 & R & R & P & P \\
508 & K & E & E & E \\
573 & E & E & A & E \\
579 & T & T & T & E \\
\hline
\end{tabular}

binding domain of VP1, implying their host adaptation and potential roles in the attenuation of GPV. Notably, compared with the GPV-CZM-70, GPVCZM-142 had 6 mutations (Q149L, E199D, A203S, L501P, R507P, E573A) in NS, 9 mutations in VP1 (Q89R, D110N, I302L, N381K, G545S, S546T, T547S, A549T, V643I) and large insertion in ITR. These additional mutations in NS and VP1 and the large insertion in ITR in the GPV-CZM-142 may contribute to the difference in virulence between GPV-CZM-70 and GPV-CZM-142.

\section{Conclusion}

In summary, a novel highly attenuated GPV strain GPVCZM-142 was generated through serial passages of the virulent strain GPV-CZM in GEF, and novel mutations related to the adaptation were identified in the genome of the GPV-CZM-142. The mutations in ITR affecting the activity of the ITR and contributing to the attenuated phenotype of the two adapted strains need to be 
Table 5 Mutations in VP1 protein of GPV-CZM-70 and GPVCZM-142

\begin{tabular}{|c|c|c|c|c|}
\hline Position & GPV-CZM & CZM-70 & CZM-142 & SYG61v \\
\hline 3 & $T$ & $\mathrm{~N}$ & $\mathrm{~N}$ & $T$ \\
\hline 5 & L & V & V & L \\
\hline 89 & Q & Q & R & Q \\
\hline 110 & $D$ & $\mathrm{D}$ & $\mathrm{N}$ & $\mathrm{D}$ \\
\hline 142 & $D$ & $\mathrm{~N}$ & $\mathrm{~N}$ & $D$ \\
\hline 146 & T & K & K & T \\
\hline 193 & $\mathrm{~T}$ & $P$ & $P$ & $P$ \\
\hline 201 & K & K & K & E \\
\hline 217 & V & L & L & V \\
\hline 302 & I & I & L & I \\
\hline 360 & $P$ & A & A & $P$ \\
\hline 381 & $N$ & $\mathrm{~N}$ & K & $\mathrm{N}$ \\
\hline 449 & S & $\mathrm{R}$ & R & $S$ \\
\hline 503 & $\mathrm{~F}$ & V & V & V \\
\hline 516 & L & $P$ & $P$ & $P$ \\
\hline 532 & K & Q & Q & Q \\
\hline 534 & I & M & M & I \\
\hline 537 & 1 & L & L & L \\
\hline 544 & S & $P$ & $P$ & S \\
\hline 545 & G & G & S & G \\
\hline 546 & S & S & T & S \\
\hline 547 & T & T & S & T \\
\hline 549 & A & A & T & A \\
\hline 555 & $\mathrm{R}$ & M & M & M \\
\hline 593 & D & $E$ & E & D \\
\hline 643 & V & V & I & V \\
\hline
\end{tabular}

confirmed by using the mini-genome system or by corresponding recombinant viruses.

\section{Methods}

Virus strains and goose embryos

A highly pathogenic GPV strain, GPV-CZM, was kept in our laboratory. From 11-day-old goose embryos without GPV maternal antibody, where the goose embryonic fibroblasts (GEFs) were isolated, were kindly provided by Dr. Zhang (Sinopharm Yangzhou VAC Biological Engineering Co. Ltd).

\section{Preparation of GEFs}

Several 11-day-old goose embryos were selected for GEF preparation. The heads, limbs, and internal organs were first removed. The remaining embryo bodies were then rinsed with PBS, followed by digestion with trypsin. After filtering with 6 layers of gauze, the GEFs were cultured in DMEM (Gibco, NY, USA) supplemented with
10\% FBS (fetal bovine serum) (Lonsera, Shanghai, China) at $37{ }^{\circ} \mathrm{C}$ with $5 \% \mathrm{CO}_{2}$.

\section{Virus passaging}

The GEFs prepared above were infected with the GPV-CZM strain (1,10 dilution in PBS) for $2 \mathrm{~h}$. After rinsed with PBS once, the infected GEFs were cultured in DMEM supplemented with 1\% FBS for 5-7 $\mathrm{d}$, followed by collection. The cells were then frozen and thawed once. The supernatants were isolated from the infected cells, followed by infecting newly isolated GEFs with it. Like this, the passaging of the virus went into the next cycle.

$\mathrm{TCID}_{50}$ assay

GEFs were infected with the viruses that were diluted with DMEM containing 1\% FBS at 1:10, 1:102, 1:103, 1: $104,1: 105$, and 1:106 in 96-well plates ( 6 wells per dilution). At day 5 post-inoculation, the cells were fixed by the ice-cold acetone: ethanol $(3,2)$ mixture for $5 \mathrm{~min}$ and washed with PBS. The cells were then stained with the diluted monoclonal primary antibody specific to GPV for $45 \mathrm{~min}$ at $37^{\circ} \mathrm{C}$. After washing three times with PBS, the cells were stained with the diluted secondary antibody (goat anti-mouse IgG-FITC) for another $45 \mathrm{~min}$ at $37^{\circ} \mathrm{C}$. Again, after three washes with PBS, the cells were observed under inverted fluorescence microscopy. The TCID $_{50}$ of these viruses was calculated by the ReedMuench method based on indirect immunofluorescence intensity.

\section{$E D_{50}$ assay}

Different GPV strains were diluted with PBS at 1:10, 1: $10^{2}, 1: 10^{3}, 1: 10^{4}$ and $1: 10^{5}$ and injected into the allantoic cavity of 12 day-old goose embryos (5 embryos for each dilution). The morbidity and mortality of the inoculated goose embryos were recorded daily.

\section{Genome sequencing analysis}

The viral DNA was isolated from different GPV strains using the DNA extraction kit and store at $-20^{\circ} \mathrm{C}$. The PCR primers based on the genome sequence of the standard strain B (U25749) were used (Table 1) to amplify the whole genome of GPV $[5,12]$. The PCR products were then cloned into the $\mathrm{PGEM}^{\circ}-\mathrm{T}$ vector (Promega) and transformed into DH5 $\alpha$ E. coli competent cells. The recombinant plasmids were extracted and sequenced by Huada Gene Technology Co., Ltd.

\section{Identification of sequence mutations}

The whole-genome sequences from different GPV-CZM strains were aligned and compared using DNAStar Lasergene software. The regions of ITR, NS, and VP1 from two replicates of GPV-CZM were further analyzed 
with GPV reference strains using MEGA6.1 software (Table 1).

\section{Abbreviations}

GPV: Goose parvovirus; GEF: Goose embryonic fibroblasts; ITR: Inverted terminal repeat; ORF: Open reading frame; NS: Non-structural protein; VP: Capsid protein; DMEM: Dulbecco's modified Eagle's medium; FBS: Fetal bovine serum; $E_{L} D_{50}: 50 \%$ Embryo Lethal Dose; $\mathrm{TCID}_{50}$ : 50\% Tissue Culture Infectious Dose

\section{Acknowledgments}

We thank Dr. Jianjun Zhang (Sinopharm Yangzhou VAC Biological Engineering Co.Ltd) for kindly providing us goose embryos without GPV maternal antibody, and thanks for Prof. Tuoyu Geng (College of Animal Science and Technology, Yangzhou University) for edition and proofreading of the manuscript.

\section{Authors' contributions}

$H S$ and $A Q$ conceived and designed the experiments. YJ, $H Y$, and $L J$ performed the experiments. YJ, AQ, KQ, WJ, and HS analyzed the data. JY, WJ, and KQ contributed reagents/materials/analysis tools. HS, YJ, and JY contributed to the writing of the manuscript. HS and YJ prepared the figures. All authors read and approved the final manuscript.

\section{Funding}

This study was supported by the National Key Research \& Development (R\&D) Plan (2017YFD0500802041), NCFC-RCUK-BBSRC (Grant No.

31761133002 and BB/R012865/1), Key Laboratory of Prevention and Control of Biological Hazard Factors (Animal Origin) for Agrifood Safety and Quality (26116120), the Priority Academic Program Development of Jiangsu Higher Education Institutions. The funding bodies did not play direct roles in the study's design and collection, analysis, and interpretation of data and writing the manuscript.

\section{Availability of data and materials}

The datasets used and/or analyzed during the current study are available from the corresponding author on reasonable request.

\section{Ethics approval and consent to participate}

Not applicable.

\section{Consent for publication}

Not applicable.

\section{Competing interests}

The authors declare that they have no competing interests.

\section{Author details}

${ }^{1}$ Key Laboratory of Jiangsu Preventive Veterinary Medicine, Key Laboratory for Avian Preventive Medicine, Ministry of Education, College of Veterinary Medicine, Yangzhou University, No.12 East Wenhui Road, Yangzhou 225009, Jiangsu, China. ${ }^{2}$ Jiangsu Co-innovation Center for Prevention and Control of Important Animal Infectious Diseases and Zoonoses, Yangzhou 225009, Jiangsu, China. ${ }^{3}$ Joint International Research Laboratory of Agriculture and Agri-Product Safety, the Ministry of Education of China, Yangzhou University, Yangzhou 225009, Jiangsu, China. ${ }^{4}$ Institutes of Agricultural Science and Technology Development, Yangzhou University, Yangzhou 225009, Jiangsu, China.

Received: 28 June 2020 Accepted: 9 November 2020

Published online: 23 November 2020

\section{References}

1. Fang DY. Introduction of "little goose plague". Chin Veterinary J. 1962;8:1920.

2. Fang DY, Wang YK, Zheng YM, Zhou YS, Jiang MJ, Dong GX. Studies on pathogens of gosling plague and their specific control. Chin Agricultural Sci. 1981;14(4):1-9.

3. Bian G, Ma H, Luo M, Gong F, Li B, Wang G, Mohiuddin M, Liao M, Yuan J. Identification and genomic analysis of two novel duck-origin GPV-related parvovirus in China. BMC Vet Res. 2019;15(1):88..
4. Yu K, Ma X, Sheng Z, Qi L, Liu C, Wang D, Huang B, Li F, Song M. Identification of Goose-Origin Parvovirus as a Cause of Newly Emerging Beak Atrophy and Dwarfism Syndrome in Ducklings. J Clin Microbiol. 2016; 54(8):1999-2007.

5. Shien J-H, Wang Y-S, Chen C-H, Shieh HK, Hu C-C, Chang P-C. Identification of sequence changes in live attenuated goose parvovirus vaccine strains developed in Asia and Europe. Avian Pathol. 2008;37(5):499-505.

6. Zhang QS, Chen-Xi LI, Liu M, Wu-Lin S, Zhang Y. Goose parvovirus attenuation and sequence analysis for the mutations in genome DNA of parental virus and its attenuated strains. Chin J Preventive Veterinary Med. 2016;6:455-7.

7. Cotmore SF, Agbandje-Mckenna M, Chiorini JA, Mukha DV, Davison AJ. The family Parvoviridae. Arch Virol. 2013;159(5):1239-47.

8. Doerig C, Hirt B, Antonietti JP, Beard P. Non-structural protein of parvoviruses B19 and minute virus of mice controls transcription. J Virol. 1990;64(1):387-96

9. Nuesch JPF, Rommelaere J. NS1 interaction with CKIla: novel protein complex mediating parvovirus-induced cytotoxicity. J Virol. 2006;80(10): 4729-39.

10. Wang JY, Huang Y, Duan JK, Zhu LQ, Zhu GQ. Amino acid point mutations in VP1 protein of goose embryo-adapted vaccine strain of goose parvovirus play a key role in its attenuation to goslings. Chin J Veterinary Sci. 2016;36: 2005-13.

11. Liu P, Yang L, Zhang J, Wang T, Wu Y, Wang M, Jia R, Zhu D, Liu M, Zhao X, et al. The 164K, 165K, and 167K residues of VP1 are vital for goose parvovirus proliferation in GEFs based on PCR-based reverse genetics system. Virol J. 2019;16(1):136.

12. He YL. Cloning and genetic evolution analysis of 5 waterfowl parvovirus genomes: Northeast Agricultural University; 2014.

\section{Publisher's Note}

Springer Nature remains neutral with regard to jurisdictional claims in published maps and institutional affiliations.
Ready to submit your research? Choose BMC and benefit from:

- fast, convenient online submission

- thorough peer review by experienced researchers in your field

- rapid publication on acceptance

- support for research data, including large and complex data types

- gold Open Access which fosters wider collaboration and increased citations

- maximum visibility for your research: over $100 \mathrm{M}$ website views per year

At BMC, research is always in progress.

Learn more biomedcentral.com/submissions 